\section{CONOCIMIENTOS DE TUBERCULOSIS EN PERSONAL DE FARMACIAS DE UN DISTRITO DE LIMA, PERÚ}

\author{
KNOWLEDGE ABOUT TUBERCULOSIS IN \\ PHARMACY PERSONNELS IN A DISTRICT OF \\ LIMA, PERU.
}

\section{Zoraida Del Solar-Moreno ${ }^{1,2, a}$, Angie Álvarez-De La Cruz ${ }^{1,2, a}$, Raúl Mendoza-Fabian ${ }^{1, b}$, Gustavo Hernández-Córdova ${ }^{1, b}$}

Sr. Editor. En el Perú existe una alta prevalencia de casos de autoprescripción y dispensación libre de medicamentos y es común encontrar que un buen porcentaje de pacientes antes de consultar a un centro de salud, acuden a las farmacias ${ }^{(1,2)}$, es así que las farmacias han sido consideradas como primer punto de contacto con los pacientes, ya que cuentan con mayor flexibilidad en horarios, ubicación y rapidez en atención $(3,4)$; esta característica ha sido utilizada por múltiples estudios para realizar diferentes intervenciones.

Entonces, es importante tener un registro acerca de los conocimientos sobre la tuberculosis en el personal de farmacias, porque al ser el primer punto de contacto de pacientes sintomático respiratorios, pueden ser importantes aliados en la prevención y diagnóstico precoz de la tuberculosis. Por ello, se realizó un estudio transversal en el distrito de Breña en el año 2015 para evaluar los conocimientos sobre la tuberculosis en personal de atención en farmacias.

Se utilizó un cuestionario estructurado aplicado por encuestadores entrenados acerca del conocimiento de tuberculosis, la encuesta constó de 18 preguntas de opción múltiple con una respuesta correcta por cada ítem. La validación del cuestionario consistió en dos etapas, en primer lugar, la validación de constructos, del cual se tomó en cuenta el formato de un cuestionario validado, usado en un estudio previo realizado el año 2013 sobre conocimientos y actitudes hacia la tuberculosis en personal de atención de los Centros de Salud de Lima, Perú (4); posteriormente, se adaptó la información a la última Norma Técnica del Ministerio de Salud del Perú sobre tuberculosis del año 2014; en segundo lugar, pasó por una revisión por dos expertos sobre el tema (un neumólogo y un salubrista).

Se escogieron 63 establecimientos de los alrededores del Hospital Nacional Arzobispo Loayza de Breña y se invitó a un trabajador de cada establecimiento a participar en el estudio. Todos los cuestionarios fueron aplicados previo consentimiento informado. De los 63 cuestionarios, tres no fueron respondidos correctamente y dos no fueron devueltos; la edad media de los participantes fue 27 años (intervalo de 19 a 72 años). Respecto al nivel de conocimientos acerca de tuberculosis el 98,4\% respondió que la enfermedad se transmite entre las personas, $49,2 \%$ respondieron que casi siempre los pacientes con tuberculosis se curan; 9,5\% respondieron que pacientes con TB-MDR serían aquellos que muestran resistencia a isoniazida y pirazinamida, y 33,3\% de los encuestados respondieron que la consecuencia del tratamiento incompleto es la tuberculosis resistente. El porcentaje de respuestas correctas fue de $55,6 \%$ (Tabla 1 ).

En nuestros resultados se evidencia que con respecto al agente infeccioso y su forma de transmisión mediante la tos hay poco porcentaje de error; sin embargo, conforme se va preguntando conceptos básicos sobre despistaje, tratamiento y algunas definiciones, el porcentaje de error va en aumento. Llama la atención que en nuestros resultados cerca del $50 \%$ conoce que la tuberculosis se pueda curar, también que aproximadamente el $65 \%$ de los encuestados no sabe que el tratamiento incompleto puede generar resistencia.

En conclusión, estos resultados pretenden contribuir sobre una problemática relevante que es la tuberculosis, sentamos un antecedente informativo en este distrito que podría ser utilizada para la prevención de contagio de esta población en riesgo, además de servir de apoyo para futuras propuestas de intervención enfocada en personal de farmacias del cual sería importante evaluar los resultados.

Conflictos de interés: los autores declaran no tener conflictos de interés en la publicación de este artículo

Financiamiento: autofinanciado

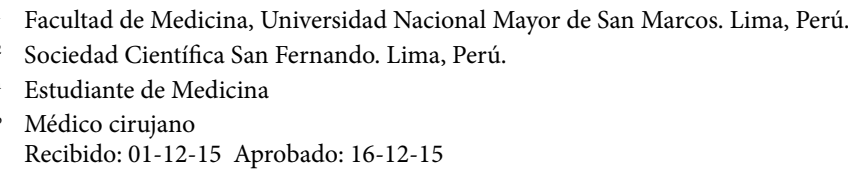


Tabla 1. Porcentajes de respuestas correctas por cada pregunta planteada

\begin{tabular}{|c|c|}
\hline Preguntas & $\begin{array}{l}\text { Porcentaje } \\
\qquad N=58\end{array}$ \\
\hline Mycobacterium tuberculosis & $74,6 \%$ \\
\hline \multicolumn{2}{|l|}{ Es transmisible entre personas } \\
\hline Sí, es transmisible de persona a persona & $98,4 \%$ \\
\hline \multicolumn{2}{|l|}{ Riesgo alto } \\
\hline En personas que tienen contacto directo con personas con tuberculosis & $50,8 \%$ \\
\hline \multicolumn{2}{|l|}{ Todos tienen síntomas } \\
\hline No todas las personas con tuberculosis presentan síntomas & $23,8 \%$ \\
\hline \multicolumn{2}{|l|}{ Síntoma más frecuente presente en personas con tuberculosis } \\
\hline Tos & $92,1 \%$ \\
\hline \multicolumn{2}{|l|}{ Examen más útil } \\
\hline Examen de esputo & $71,4 \%$ \\
\hline \multicolumn{2}{|l|}{ Numero de muestras necesarias para examen de esputo } \\
\hline Dos muestras para examen de esputo & $36,5 \%$ \\
\hline \multicolumn{2}{|l|}{ Duración del tratamiento para tuberculosis sensible } \\
\hline Seis meses de tratamiento & $61,9 \%$ \\
\hline \multicolumn{2}{|l|}{ Como defines a una persona con TB MDR } \\
\hline Persona que muestra resistencia a isoniazida y pirazinamida & $9,5 \%$ \\
\hline \multicolumn{2}{|l|}{ Consecuencia de un tratamiento inadecuado } \\
\hline TBC resistente & $33,3 \%$ \\
\hline \multicolumn{2}{|l|}{ El mejor tratamiento para tuberculosis } \\
\hline Es el tratamiento indicado por el centro de salud & $79,4 \%$ \\
\hline \multicolumn{2}{|l|}{ Se cura la tuberculosis } \\
\hline Casi siempre & $49,2 \%$ \\
\hline
\end{tabular}

\section{REFERENCIAS BIBLIOGRÁFICAS}

1. Fathima M, Naik-Panvelkar P, Saini B, Armour CL. The role of community pharmacists in screening and subsequent management of chronic respiratory diseases: a systematic review. Pharm Pract (Granada). 2013;11(4):228-45.

2. Hersberger KE, Renggli VP, Nirkko AC, Mathis J, Schwegler K, Bloch KE. Screening for sleep disorders in community pharmacies-evaluation of a campaign in Switzerland. J Clin Pharm Ther. 2006;31(1):35-41. doi: $10.1111 / j .1365-2710.2006 .00698 . x$

3. Snella KA, Canales AE, Irons BK, Sleeper-Irons RB, Villarreal MC, Levi-Derrick VE, et al. Pharmacy-and community-based screenings for diabetes and cardiovascular conditions in high- risk individuals. J Am Pharm Assoc (2003). 2006;46(3):3707. doi:10.1331/154434506777069598

4. Minnery M, Contreras C, Pérez R, Solórzano N, Tintaya $\mathrm{K}$, Jimenez J, et al. A cross sectional study of knowledge and attitudes towards tuberculosis amongst front-line tuberculosis personnel in high burden areas of Lima, Peru. PLoS One. 2013;8(9):e75698. doi: 10.1371/journal.pone.0075698

Correspondencia: Zoraida Del Solar Moreno

Dirección: $M z$ G lote 1 Av. Los dominicos

Teléfono: (511) 969707867

Correo electrónico:zori26dsm@hotmail.com 